\title{
La coopération économique entre la République Démocratique du Congo, le Rwanda et le Burundi : état des lieux et perspectives.
}

\author{
Par Camille NGOMA KHUABI ${ }^{1}$
}

\begin{abstract}
Résumé
La RDC, le Burundi et le Rwanda ont su développer des relations amicales fondées sur des traités de coopération bilatérale sans mécanisme de mise en œuvre. La création de la Communauté Économique des Pays des Grands Lacs (CEPGL) marquait l'institutionnalisation d'une coopération économique qui a quelque peu profité aux trois pays membres. L'histoire démontre toutefois que cette organisation n'a pas été à la hauteur de la conjoncture socio politico-économique de ses États membres. La relance de la coopération économique actuellement encouragée entre ces trois pays s'inscrit dans une perspective de consolidation de la paix dans cette phase post-conflit. Elle devrait allier exploitation commune des ressources naturelles transfrontalières, paix, stabilité régionale, et impératifs démocratiques des États impliqués.
\end{abstract}

\begin{abstract}
The DRC, Burundi and Rwanda knew to develop friendly relations based on treaties of bilateral cooperation without mechanism of implementation. The creation of the Economic Community of the Countries of Great Lakes (CEPGL) marked the institutionalization of an economic cooperation which somewhat benefitted the three member countries. The history shows however that this organization was not worthy of the politico-economic situation socio of its member states. The revival of the economic cooperation currently encouraged between these three countries falls under a prospect for consolidation of peace in this phase post-conflict. It should combine common exploitation of the transborder natural resources, peace, regional stability, and democratic requirements of the implied States.
\end{abstract}

\section{INTRODUCTION}

La République Démocratique du Congo (RDC) est un pays du centre de l'Afrique entouré par neuf autres États appartenant à différentes communautés économiques régionales. Cette position fait de ce pays un véritable espace géographique ouvert à des interconnexions qui l'ont conduit, d'abord, dans un réseau d'accords de coopération bilatérale entre chacun de

1 Chercheur et doctorant en droit international public, UNIKIN/RDC, E-mail : camillengomak@gmail.com. 
ses voisins, ensuite, dans le cadre des organisations d'intégration économique sous régionales, dont la Communauté Économique des Pays des Grands Lacs (CEPGL) dans laquelle il est associé au Rwanda et au Burundi, deux pays de l'Est de son territoire.

L'intérêt marqué pour la RDC, à l'origine, à la coopération avec ces deux pays pourrait actuellement être évalué à la lumière des enjeux de la crise qui l'a secoué, dès la fin du règne du Président Mobutu, crise qui s'est cristallisée dans sa partie orientale, directement frontalière avec ses deux derniers voisins. D'aucuns ont pu même soutenir que les guerres qui ont éclaté dans la région des Grands Lacs sont des conséquences de l'échec de la CEP$\mathrm{GL}^{2}$.

Aussi, examiner la question de la coopération économique entre la RDC et ses deux voisins membres de la CEPGL s'analyse dans la perspective de sortie de cette crise, tant il est vrai, qu'une véritable coopération, fondée sur une idée d'intégration économique des populations des trois pays recréerait un climat de confiance rompue par deux décennies de conflit. Sans cela, le difficile processus actuel de reconstruction d'une paix fondée sur une multitude des résolutions ${ }^{3}$ et d'accords, ne s'appuierait que sur des fondements fragiles.

Relancer une coopération économique en perspective (B) revient, dans un premier temps, à présenter ses acquis sur lesquels peuvent se greffer les idées actuelles tendant à recréer une plus grande intégration des populations par le développement des projets transfrontaliers. Il sera très intéressant d'inventorier ceux-ci dans un second temps(A) afin de présenter le potentiel économique de l'espace considéré.

\section{A. LES ACQUIS DE LA COOPÉRATION ENTRE LA RDC ET SES DEUX VOISINS.}

Au cours de trente dernières années ayant précédé les indépendances de ces trois pays, la coopération entre ces derniers s'est d'abord construite autour des accords bilatéraux (A), ensuite dans le cadre de la CEPGL (B).

\section{Les Accords bilatéraux : une coopération intergouvernementale classique}

Il s'agit, à ce stade, de présenter les grandes lignes de quelques accords ayant lié la RDC et chacun de ses deux voisins, mais, dont la mise en œuvre n'aura parfois connu aucun début de réalisation faute d'organes permanents de mise en œuvre. Les identifier simplement d'abord donnerait plus de lumière à la perspective d'une coopération plus efficace. Dans le cadre d'une analyse plus approfondie, l'on travaillerait à leur amélioration.

2 Conférence Internationale sur la Région des Grands Lacs, Programme d'Action Régional pour le Développement Économique et Intégration Régionale, Relance de la Communauté Économique des Pays des Grands Lacs et de ses institutions spécialisées EGL, SINELAC, IRAZ, et BDEGL, 2006, p.2.

3 Adoptées notamment du Conseil de Sécurité des Nations Unies dès le début de la crise entre ces trois pays, le 2 août 1998. 
1. La Convention entre la République démocratique du Congo et la République Rwandaise

Dans le cadre de cette Convention signée à Léopoldville (Kinshasa actuel), le 4 avril 1966, les deux États désirent assurer, sur la base de la réciprocité et dans les meilleures conditions, leurs communications routières communes vers le Lac Tanganyika ${ }^{4}$. Le texte adopté à cet effet se fixe entre autres objectifs: la conservation et le maintien en bon état de la route asphaltée qui relie Bukavu-Bugarama au Lac Tanganyika, via Kavimvira, ainsi que de son libre usage pour le transit des marchandises ${ }^{5}$ et des personnes autres que les militaires en armes $^{6}$. Aux termes de son article premier, les tronçons de la route du côté de la RDC sont délimités comme suit : (i) Bukavu-pont sur la Ruzizi, près de Bukavu; (ii) Pont sur la Ruzizi, près de Bugarama-Kavimvira; (iii) Kavimvira-pont sur la petite Ruzizi, frontière du Burundi, vers Usumbura; (iv) Kavimvira-Kalundu sur le Lac Tanganyika. Le tronçon de la route se trouvant sur le territoire rwandais part du pont sur la Ruzizi, près de Bukavu-pont, près de Bugarama.

2. Traité d'amitié et de coopération entre la République du Zaïre et la République Rwandaise.

Ce Traité a été conclu à Kigali, au Rwanda, le 28 octobre 1972. Il entend favoriser une coopération dans tous les domaines. Les États devraient y parvenir en promouvant ensemble des projets répondant à un intérêt commun, en vue de leur avantage mutuel (art.1). L'article $4 \mathrm{du}$ Traité prévoit l'organisation des consultations et des rencontres régulières entre les deux parties, de manière à leur permettre d'une part, d'étudier tout problème qu'elles jugent important et à veiller à l'avancement rapide de leurs projets d'intérêt commun, et d'autre part, à favoriser la stabilité des relations amicales entre les deux pays.

3. Accord relatif aux transports aériens entre la République démocratique du Congo et la République Rwandaise.

Cet Accord conclu à Kinshasa, le 11 septembre 1970, en vue de favoriser le développement des transports aériens et de renforcer la coopération internationale dans ce domaine entre les deux pays s'inspire des principes et dispositions de la Convention relative à l'Aviation Civile Internationales signée à Chicago le 7 décembre 19447. Plus de quarante après sa signature, la réalité démontre plutôt qu'aucune liaison aérienne n'existe encore entre ces deux pays. Même si Kigali, la capitale rwandaise se trouve à quelques minutes de la ville congo-

4 Préambule de la convention entre la République démocratique du Congo et la République Rwandaise, Léopoldville, le 4 avril 1966.

5 Idem.

6 Art. 3 de la convention entre la RDC et le Rwanda, op.cit.

7 Préambule de l'Accord relatif aux transports aériens entre la République démocratique du Congo et la République Rwandaise, Kinshasa, le 11 septembre 1970. 
laise de Goma, il peut paraître gênant de relever, par exemple que, pour atteindre cette ville depuis Kinshasa par avion, l'on doit dans certains cas passer par Addis-Abeba, en Éthiopie ou par Nairobi au Kenya. L'application de cet accord permettrait d'éviter un tel exercice à la fois couteux et fatiguant.

4. Convention de coopération entre la République du Zaïre et la République Rwandaise pour l'exploitation, le transport et la commercialisation du gaz méthane du Lac Kivu

Le Lac Kivu contient environ 300 milliards de mètres cube de dioxyde de carbone (Co2) et de 60 milliards de mètres cube de gaz méthane $(\mathrm{CH} 4)$. Selon les estimations des spécialistes, 120 à 250 millions de $\mathrm{m} 3$ de CH4 sont générés annuellement dans le lac. Le Rwanda souhaite utiliser cette ressource pour développer des projets de production d'énergie à partir du gaz méthane ainsi que pour d'autres usages tels que des projets de fabrication d'engrais et des projets gaz-liquide. Les études démontrent que le gaz méthane présent dans le Lac Kivu est suffisant pour générer 700MW d'électricité sur une période de 55 ans $^{8}$. Les deux pays se sont convenus dans un premier temps de développer conjointement $200 \mathrm{MW}$.

Dans le cadre de la convention conclue à Bukavu, le 3 mai 1975, les deux États ont décidé de créer une société commune à laquelle ils confèrent le monopole sur toutes les activités liées à l'exploitation, au transport et à la commercialisation du gaz méthane du Lac Kivu (Article 1). Chacune des parties participe à raison de $50 \%$ dans le capital social de la société à créer (Article 2). Les Parties contractantes avaient prévu de confier à l'Association «ASSINEZ » la mission d'étudier, avec des partenaires désignés par chacune d'elles la faisabilité d'une usine d'exploitation du gaz méthane, d'inventorier et d'analyser l'ensemble des problèmes relatifs au lancement d'une telle usine et d'en proposer des solutions adéquates (Article 3).

Quarante ans après sa signature, les conditions matérielles de sa mise en œuvre semblent se préciser, puisque, le 19 novembre 2015, en prévision du lancement des activités de l'exploitation du gaz méthane, les deux pays ont signé à Gisenyi, au Rwanda, un autre accord portant essentiellement sur la surveillance de ce cours d'eau au moment des travaux d'exploitation du gaz ${ }^{9}$. L'installation, ce 27 avril 2016, d'un comité mixte ${ }^{10}$ de 10 experts chargé de la surveillance de l'exploitation du gaz méthane dans ce lac constitue, en effet, une autre étape franchie pour la mise en œuvre effective de cette coopération.

8 Document sur les Opportunités d'Investissement (DOI), processus de consultation, leçons apprises et principales conclusions, p.28.

9 La RDC et le Rwanda signent un accord en prévision de l'exploitation du lac Kivu, [en ligne] http://www.radiookapi.net/2015/11/20/actualite/societe/la-rdc-et-le-rwanda-signent-un-accord-enprevision-de-lexploitation-du, ce 20 avril 2016.

10 RDC-Rwanda : un comité pour surveiller l'exploitation du gaz dans le lac Kivu, [en ligne] http://w ww.radiookapi.net/2016/04/28/actualite/societe/rdc-rwanda-un-comite-pour-surveiller-lexploitatio n-du-gaz-dans-le-lac, le 20 avril 2016. 
À notre sens, ce comité devrait avoir pour mission, outre la surveillance des activités, celle d'examiner, de rejeter et/ou de valider les rapports des études d'impact environnemental effectuées par les sociétés exploitantes. Le comité se doterait en ce sens de l'expertise et des moyens humains, matériels et financiers nécessaires à la hauteur de ses missions.

5. Accord commercial entre la République du Burundi, la République Rwandaise et la République du Zaïre.

Ce dernier Accord a été conclu à Kigali, le 21 juin 1975 entre les trois États. Son analyse démontre qu'il s'inscrit dans le cadre du programme de la réalisation progressive d'une communauté économique entre ces trois pays ${ }^{11}$. Son objectif est de promouvoir et d'intensifier les échanges entre les Parties. Aux termes de son article $1^{\text {er }}$, les Parties s'engagent mutuellement à autoriser, sans formalités que celles de l'introduction d'une demande de licence d'importation et d'exportation, par les soins de l'importateur et de l'exportateur intéressé, l'exportation ou l'importation des marchandises figurant aux listes A, B et C annexés au présent Accord et produites sur leurs territoires respectifs.

Il résulte de la lecture de ces différents accords que la coopération est restée au niveau intergouvernemental, où des commissions mixtes d'experts non permanentes devraient se réunir sporadiquement en vue d'évaluer une politique et proposer des options pour la mise en œuvre. Seule la Convention de coopération entre la République du Zaïre et la République Rwandaise pour l'exploitation, le transport et la commercialisation du gaz méthane du Lac Kivu avait formellement prévu la création d'une Société commune qui devrait symboliser une coopération plus réelle dans un domaine tout aussi vital pour les États. Cette société n'avait jamais vu le jour. Les nécessités sociales de deux peuples démontrent plutôt que les deux pays sont dans une obligation liée de coopérer.

Dans le cadre de la coopération envisagée actuellement et, dans la perspective d'une sortie définitive de crise, il est important d'évaluer l'ensemble des initiatives prises en vue de déterminer les priorités et les mesures à prendre à court, à moyen et à long terme. Car, le rétablissement d'un climat propice au développement d'une coopération saine entre ces pays doit être assis sur une base sociale. Les projets à développer actuellement devront davantage s'attaquer aux secteurs qui peuvent regrouper les populations de deux ou de ces trois pays.

\section{La RDC dans la CEPGL : une coopération économique institutionnalisée}

La CEPGL est une organisation internationale sous régionale réunissant les trois pays frontaliers, le Burundi, le Rwanda et la RDC, tous, anciennes colonies belges. Créée le 20 sep-

11 Préambule de l'Accord commercial entre la République du Burundi, la République Rwandaise et la République du Zaïre, Kigali, le 21 juin 1975. 
tembre 1976 à Gisenyi ${ }^{12}$, au Rwanda, elle tire ses origines dans les liens politico-administratifs et économiques qui unissaient ces trois pays depuis l'époque coloniale. En effet, alors que le Congo, propriété privée de Léopold II devenait en 1908 une « colonie belge ${ }^{13}$ », la Belgique avait reçu le 31 Août 1923 de la Société des Nations, le mandat sur le RuandaUrundi. En vertu de la loi sur le gouvernement du Ruanda-Urundi, le territoire sous mandat est administrativement rattaché au Congo belge le 25 août $1925^{14}$. Ce mandat fut transformé en tutelle par l'Acte des Nations Unies du 13 décembre 1946.

Sur le plan économique, le Congo Belge et le Ruanda-Urundi constituaient un espace économique intégré sous l'autorité du Gouvernement belge dans le cadre d'une union économique et monétaire de fait et opéraient des échanges de toutes sortes, sans compter les activités économiques communes et les services publics communs. Dans les lignes qui suivent, nous présentons brièvement les objectifs et les institutions de la CEPGL (1) avant de tenter d'esquisser un bilan de ses activités jusqu'au moment où se déclenche la guerre à l'origine du génocide rwandais en 1994 (2).

1. Objectifs de la convention et cadre institutionnel

Aux termes de l'article 2 de la Convention créant la CEPGL ${ }^{15}$, cette dernière a pour objectifs :

"Assurer d'abord et avant tout la sécurité des États membres et de leurs populations de façon qu'aucun élément extérieur ne vienne troubler l'ordre et la tranquillité à leurs frontières respectives; Promouvoir et intensifier les échanges commerciaux et la libre circulation des personnes et des biens; Concevoir, définir et favoriser la création et le développement d'activités d'intérêts communs; Coopérer d'une façon étroite dans les domaines social et économique, commercial, scientifique et touristique plus spécialement en matière juridique, douanière, sanitaire, énergétique, de transport et de télécommunications ».

12 Convention portant Création de la Communauté Économique des États des Grands Lacs (C.E.P.G.L), Journal officiel de la République du Zä̈re, $n^{\circ} 20$ du 15 octobre 1980, pp.5-9.

13 Dans son ouvrage sur les Aspects juridiques des crises congolaises. De l'origine à nos jours (1860-2006), publié aux éditions PUK, à Kinshasa, en 2011, le professeur Banza Malale soutient que l'actuelle RDC n'a jamais été une colonie belge puisque son territoire a évolué du statut d'Association internationale du Congo pour devenir dès 1885, à l'issu de la conférence de Berlin, un État indépendant du Congo (EIC) avant d'être confié à la Belgique en 1908 pour y assurer les actes de simple administration pour le compte de toute la communauté internationale. Il démontre par ailleurs que la constitution belge de 1830 était incompatible avec le statut de puissance coloniale injustement attribué à la Belgique. Pour des détails intéressants, voir les pp. 131-135.

14 Gahama Joseph, Le Burundi sous administration belge : la période du mandat 1919-1939, Karthala, Paris, 2001, p.44.

15 Convention portant Création de la Communauté Économique des États des Grands Lacs, op.cit. 
La CEPGL devrait finalement aboutir à la construction d'un espace économique intégré qui résulterait de l'intensification des échanges avec la suppression des obstacles internes, pour ensuite introduire progressivement des mécanismes de coordination des politiques ${ }^{16}$. Le cadre institutionnel mis en place répondait à cet objectif. Pour y parvenir, l'Organisation s'est dotée des organes ${ }^{17}$ permanents et non permanents. Le Secrétariat Exécutif Permanent, l'unique institution permanente de la communauté, a son siège Gisenyi, au Rwanda à $6 \mathrm{Km}$ de la ville de Goma en RDC. La Conférence des Chefs d'État se réunit une fois par an; le Conseil des ministres réunit les ministres des affaires étrangères des pays membres. La Commission d'Arbitrage, un organe non permanent ${ }^{18}$, devrait assurer le respect du droit dans l'interprétation et l'application de la Convention ${ }^{19}$.

\section{Bilan de la CEPGL entre 1976 et 1994}

Au cours de cette période, la CEPGL a mis en place quatre organismes spécialisés devant lui permettre de réaliser ses missions : l'organisation de la CEPGL pour l'Énergie des Grands Lacs (EGL); la Société Internationale pour l'Électricité des Grands Lacs (SINELEC); la Banque de Développement des États des Grands Lacs (BDEGL); et l'Institut de Recherche Agronomique et Zootechnique (IRAZ). Quelles sont les missions attribuées à chacun de ces organismes?

\section{a) La BDEGL}

Créée en 1977, la BDEGL qui a son siège à Goma en RDC, a pour objectif principal de mobiliser des ressources financières en vue de financer des projets dont l'objet est la poursuite de l'intégration économique et le développement de la sous région. La BDEGL s'est alors impliquée dans le financement de plusieurs projets sous régionaux. Parmi les plus importants, l'on note l'implantation d'une centrale autonome de production d'oxygène nécessaire notamment dans la fabrication d'ampoules à incandescence (Lengstram) à Kinshasa; la construction et l'équipement d'une usine textile en cofinancement avec la Banque Rwandaise de Développement (Utexrwa) à Kigali; la construction de l'hôtel diplomate à Kinshasa; la modernisation et l'extension de la sucrière de Kiliba (Sucki) en RDC; l'alimentation énergétique des relais hertziens de Nyabibwe, Kamashuli, Tshwate, Tshamata et Thomohini; la construction de la Centrale Ruzizi II - cofinancement à Bukavu; la production de tôles ondulées (Tolinki) à Bukavu; la production de peinture (Langi-Congo) à Kinshasa; l'extension d'une usine de production de cigarettes (BAT) à Kinshasa; la création de la société de

16 Alain Léon, Ronan PORHEL, « La gestion de la multi-appartenance, une nécessité pour poursuivre la stratégie régionale dans l'Afrique des Grands lacs ", Observatoire des Grands Lacs en Afrique, Note $\mathrm{n}^{\circ} 3$, juin 2012, p.5.

17 Article 5 de la Convention créant la CEPGL.

18 Selon le sens de l'article 24, alinéa 3, de la Convention créant la CEPGL.

19 Article 25 de la Convention créant la CEPGL. 
transport sur le lac Tanganyika (Arnolac) Bujumbura; la fabrication de sacs en polypropylène (Sonaco) à Bujumbura; la création de la scierie de bois (Bakri bois corporation) à Kinshasa $^{20}$. Dans une perspective de relance et de rétablissement de confiance mutuelle entre les trois pays, il est nécessaire qu'un état des lieux de l'ensemble de ces projets soit tenu. Il permettrait de soutenir et d'appuyer les initiatives qui se sont maintenues dans les trois pays.

\section{b) L'Institut de Recherche Agronomique et Zootechnique (IRAZ)}

Créé en 1979, l'IRAZ a pour mission principale de faire de la recherche en matière agronomique et zootechnique et d'intervenir dans l'exécution des projets communautaires. L'IRAZ travaille étroitement avec les Instituts nationaux de recherche agronomique pour promouvoir l'autosuffisance alimentaire au sein de la sous région. Il a son siège à Gitega au Burundi. L'IRAZ a concentré ses programmes de recherches sur la banane, les ressources phylogénétiques, les plantes à racines et tubercules, les ruminants, l'agro-climatologie, ainsi que sur la réalisation d'un centre de documentation, d'information et de formation agri$\operatorname{coles}^{21}$. Dans un contexte actuel de changement climatique ${ }^{22}$, l'IRAZ travaillerait à développer des variétés de semences plus résilientes aux impacts de la chaleur. Ici également, un état des lieux s'impose.

\section{c) L'Énergie des Grands Lacs (EGL)}

Créée en 1974 comme une ASBL, l'EGL a été intégrée dans la CEPGL en 1979. Son siège est établi à Bujumbura au Burundi. Sa mission principale est d'assurer la coopération entre les États membres dans le domaine de l'Énergie. Il devrait jouer le rôle d'organe de planification, d'études et de réalisation des projets. Il a à ce titre activement participé au processus à la base de la construction de la centrale communautaire de la Ruzizi II, dont la puissance actuelle est de 43,2 MW.

20 Conférence Internationale sur la Région des Grands Lacs, Programme d'Action Régional pour le Développement Économique et Intégration Régionale, Relance de la Communauté Économique des pays des grands lacs et de ses institutions spécialisées, Projet No. 3.2.1, 2006, pp.3-4.

21 Conférence Internationale sur la Région des Grands Lacs, Relance de la Communauté..., op.cit., p.3.

22 Sur l'impact des changements climatiques au Rwanda et Burundi notamment, lire, Ngoma Khuabi, « Dégradation de l'environnement, épuisement des ressources naturelles, explosion démographique et changements climatiques : menace sur les frontières? Regard sur la « doctrine Nguyandila », in NDESHYO RURIHOSE (Dir.), La République démocratique du Congo : Les défis récurrents de décolonisation, de l'état de droit et du développement, Mélanges au Professeur Célestin Nguyandila, CEDESURK, Kinshasa, 2012, pp.95-156. 


\section{d) La SINELAC}

Créée en 1989, la SINELAC a pour mission d'exploiter la centrale hydroélectrique communautaire de la Ruzizi II et d'assurer la commercialisation de l'énergie produite à travers les trois pays membres. Cette activité se réalise par le biais de chacune des sociétés nationales d'électricité, à savoir la Société Nationale d'Électricité (SNEL) pour la RD Congo, la REGIDESO pour le Burundi et l'ELECTROGAZ pour le Rwanda. Son siège est établi à Bukavu en RDC. Entre 1991 et 2001, la SINELAC a fourni, en moyenne, respectivement $45 \%$, $17 \%$ et $21 \%$ de la production nationale de l'énergie électrique dans le système interconnecté de la CEPGL, destinée à ces trois pays ${ }^{23}$. Tel pourrait être le bilan de la CEPGL dans le domaine d'intégration économique. D'autres efforts de la CEPGL concernent les aspects commerciaux et la libre circulation des personnes.

\section{e) La circulation des personnes, des biens et des capitaux dans la CEPGL}

Les pays de la CEPGL ont signé plusieurs instruments de coopération et d'intégration régionales portant sur des domaines tels que les échanges commerciaux, les finances (arrangement monétaire CEPGL) et la libre circulation des personnes, marquée par la mise en place d'une carte spéciale de circulation CEPGL et d'un passeport CEPGL. Si entre le Rwanda et la RDC les faits semblent aller dans ce sens, la frontière de ces deux pays entre Goma et Gisenyi étant ouverte 24h/24, il faut tout de même souligner que les efforts tendant à libéraliser la circulation des personnes, des biens et des capitaux doivent se poursuivre entre les trois pays.

En effet, cette question est chaque fois inscrite dans les agendas des dirigeants de ces pays, mais son processus n'a jamais abouti. Commencé en 1980 par la signature, le 7 décembre, d'un Arrangement entre les trois pays relatif à la libre circulation des fonctionnaires et des hommes d'affaires ressortissants des États membres de la CPGL, une carte de libre circulation CEPGL fut instituée au profit de ces derniers. Fonctionnaires et hommes d'affaires pouvaient dès lors accéder sans visa dans l'espace de l'un ou l'autre État membre. Cette mesure devait s'étendre par la suite aux étrangers dirigeant les entreprises communes ou communautaires de la CEPGL.

Le $1^{\text {er }}$ décembre 1985, un Protocole portant libéralisation du commerce des produits du cru originaires de la CEPGL fut adopté. Dans la suite de cette démarche, une Convention sur la libre circulation des personnes, des biens, des services, des capitaux et sur le droit d'établissement dans la CEPGL fut signée à Gisenyi, au Rwanda. Le Rwanda l'a aussitôt ratifiée; mais la Convention n'est jamais entrée en vigueur faute d'avoir réuni les ratifications requises, la RDC et le Burundi s'y étant opposés. Il semble que des raisons politiques

23 V. Conférence Internationale sur la Région des Grands Lacs, Programme d'Action Régional pour le Développement Économique et Intégration Régionale, Projet No. 3.2.1 Relance de la Communauté Économique des pays des grands lacs et de ses institutions spécialisées, juin 2006 (Rev. 30 août 2006), op.cit, p.4. 
et économiques expliquaient encore les réticences ${ }^{24}$ des deux pays quant à la ratification de ce précieux instrument de coopération.

Dans une perspective de sortie de crise, un autre projet de convention fut proposé aux États en 2010. Ce dernier expurge le terme « droit d'établissement » contenu dans la première non ratifiée. La différence est à rechercher dans l'esprit des initiateurs de ces projets.

En effet, pour la convention de 1985,

la liberté de circulation et le droit d'établissement impliquait, pour les ressortissants de chaque État membre, le droit d'entrée, de séjour, de résidence, de s'établir et de revenir dans les autres États membres ainsi que d'y exercer toutes les activités sociales et économiques, à l'exception des fonctions considérées normalement comme impliquant des responsabilités politiques et administratives dans le cadre de l'exercice de l'autorité publique ou aux cas où des restrictions sont strictement nécessaires dans l'intérêt de l'État.

Pour le projet de 2010 par contre,

La liberté de circulation comporte, pour les ressortissants de chaque État membre, le droit d'entrée sans visa, de séjourner, de résider, de s'établir et de revenir ainsi que d'exercer toutes les activités économiques et d'avoir accès à l'emploi et à l'enseignement primaire, secondaire et supérieur et autres formations techniques ou stages dans les conditions prévues par la présente Convention.

Nous ignorons l'état actuel du processus devant conduire à la ratification de ce nouvel instrument sous régional. Nous pouvons par contre rappeler que, en RDC, la ratification obéit à une procédure parlementaire. La question de relations entre ce pays et le Rwanda ayant toujours été traitée avec passion, les députés ayant chaque fois mis en avant l'argument des plaies encore béantes d'une agression armée, des massacres de nos frères et sœurs, et de pillage de nos ressources naturelles par les deux autres pays membres de la CEPGL, l'on peut parier que cette Convention subisse le même sort que sa devancière de 1985. Dans la perspective d'une relance économique impliquant l'extension des échanges entre populations des pays de la région, un effort de sensibilisation et de plaidoyer devrait être mené en vue d'obtenir la ratification de cette nouvelle Convention par les trois États.

Car, le problème paraît plus complexe que l'on ne pense. En effet, le Burundi, mais surtout le Rwanda ${ }^{25}$, connaissent une situation démographique exponentielle à côté d'un grand

24 CIFENDE KACIKO Moïse, "Vers la ratification d'une convention sur la libre circulation des personnes, des biens, des services et des capitaux dans la Communauté des Pays des Grands Lacs? », in NDESHYO RURIHOSE (Dir.), La République démocratique du Congo : les défis récurrents de décolonisation, de l'État de droit et du développement économique et social, Mélanges Célestin NGUYA-NDILA, CEDESURK, Kinshasa, 2012, p.318.

$25 \mathrm{Au}$ début du $20^{\mathrm{ème}}$ siècle, la population rwandaise était estimée à 1 million d'habitants. En 1991, elle atteignait 7 millions et les projections des Nations Unies avançaient le chiffre de 20 millions 
Congo sous peuplé26. Au Burundi comme au Rwanda, les déficits de production agricole, dans un contexte de dégradation de l'environnent et de pénuries des zones agricoles, a été chaque fois à l'origine des violences ethniques dont la forme la plus cruelle s'est manifestée dans le drame génocidaire connu notamment au Rwanda en 199427. Et comme le fait remarquer Roland Pourtier, dans un contexte technique d'exploitation du milieu, la capacité de charge est souvent dépassée, contraignant une fraction de la population à l'émigration ${ }^{28}$. Puisque la question démographique ne peut pas être éludée, il semble que dans ce pays, et dans des situations de plus en plus fréquentes, le choix se pose crûment entre le préservatif et la kalachnikov ${ }^{29}$.

Conscients de cet état des choses et notamment la bipolarité ethnique à l'origine des conflits entre les deux principales ethnies au Rwanda et au Burundi, des intellectuels hutus rwandais ont dû élaborer, en 1995, un projet proposant carrément l'annexion du Rwanda à la RDC ou à la Tanzanie ${ }^{30}$, alors que Ignacio Ramonet proposait de revenir à l'idée des États-Unis d'Afrique centrale ${ }^{31}$. La première comme la seconde hypothèse ne nous paraît pas trop exagérer, car, si nous devons considérer les prévisions démographiques actuelles de ce pays, il y a logiquement lieu de s'interroger avec Roland Pourtier, comment le Rwan$\mathrm{da}$, encore essentiellement agricole pourra-t-il y faire face?

Le bilan ainsi présenté ne veut nullement dire que la CEPGL a atteint ses objectifs. Il veut simplement renseigner que, au-delà de nombreuses difficultés qui ont entravé son fonctionnement, cette Organisation a réellement fonctionné dans un contexte de paix, et peut encore servir de moteur pour une coopération qui devra s'étendre à moyen et à long terme, à l'ensemble des États de l'actuelle CIRGL. Plusieurs observateurs penchent en ce sens. La CIRGL a d'ailleurs servi de laboratoire d'un projet de plaidoyer en faveur de la relance de cette Organisation.

d'habitants pour 2025 avant de revoir ces prévisions à la baisse soit, 22 millions d'habitants en 2050 .

26 Selon les estimations non officielles, la RDC compte actuellement 70 millions d'habitants pour un espace cinq fois plus vaste que la France qui, elle, abrite une population de 60 millions d'habitants sans être dans une situation de pénurie de terres agricoles. Comparativement à son espace territorial, la RDC peut raisonnablement abriter 300 millions d'habitants sans se plaindre de déficits des terres agricoles.

27 Pour des détails sur le lien entre dégradation de l'environnement et conflit, lire Ngoma Khuabi (C), « Dégradation de l'environnement », op.cit.

28 Roland Pourtier, Afrique Noires, $2^{\text {ème }}$ édition revue et augmentée, Hachette, 2010, ISBN : 987-2-01-146141-4, p.53.

29 Idem.

30 Mabiala mantuba ngoma, «La guerre d'agression de la RDC (1999-2002) : évolution, perceptions et perspectives, in Labana Lokwa (B), La prévention des crises et l'instauration d'une paix durable en RDC, publication de l'Institut pour la Démocratie et le Leadership Politique, Kinshasa, 2002, p. 35.

31 Idem. 


\section{B. PERSPECTIVES DE LA COOPÉRATION ÉCONOMIQUE ENTRE LA RDC LE BURUNDI ET LE RWANDA}

La coopération entre la RDC et ses voisins est actuellement encouragée par la Conférence Internationale pour la Région des Grands Lacs (CIRGL), et encadrée par le dernier Accordcadre du 24 février 2013, pour la paix, la sécurité et la coopération pour la République démocratique du Congo et la Région. La CIRGL est une structure qui résulte du Pacte pour la Sécurité, la Stabilité et le développement dans la région des Grands Lacs adopté à Nairobi en 2006. Ce document énonce plusieurs Programmes intégrateurs dont la réalisation passe nécessairement par la mise en œuvre des projets impliquant directement les populations de la RDC et ses deux voisins de la CEPGL. De même, l'Accord-cadre d'Addis-Abeba entend accompagner le processus de paix en soutenant en RDC des actions concrètes ${ }^{32}$, qui favorisent l'approfondissement de l'intégration économique ${ }^{33}$ résultant d'une coopération régionale ${ }^{34}$ entre ce pays et ses voisins.

Nous présentons, à la lumière des documents disponibles, quelques idées des projets formulés dans le cadre de la CIRGL (A), ceux devant être financés dans le cadre des engagements pris par la Communauté internationale au titre de l'Accord-cadre pour la paix, la sécurité et la coopération pour la République démocratique du Congo et la Région (B), d'autres projets pouvant provenir du secteur privé $(C)$. À travers cette présentation, la RDC aura à opérer le choix selon ses propres priorités de développement.

\section{Projets identifiés dans le cadre de la CIRGL}

Le Pacte pour la Sécurité, la Stabilité et le Développement dans la région des Grands Lacs comporte quatre Programmes complétés par d'autres protocoles. La mise en œuvre de chacun de ces programmes se traduit par la réalisation des projets d'intégration. La CIRGL a pu élaborer quelques-uns dont nous présentons quelques idées maîtresses.

1. Projet de Relance de la Communauté Économique des Pays des Grands Lacs et de ses institutions spécialisées EGL, SINELAC, IRAZ, et BDEGL ${ }^{35}$.

Après plusieurs années de conflit, la relance de la coopération et de l'intégration économiques dans le cadre de la CEPGL apparaît comme le cadre le plus indiqué aux États membres de trouver des solutions aux problèmes qui se posent non seulement pour le développement économique et social, mais aussi pour la restauration de la paix et de la sécurité

32 Point 4, de l'Accord-cadre pour la paix, la sécurité et la coopération pour la République démocratique du Congo et la Région, 24 février 2013, p.1.

33 Accord-cadre pour la paix, op.cit., p. 3.

34 Idem.

35 Ibid. 
dans la sous-région ${ }^{36}$. Chacun de ces organes techniques de la CEPGL analysés plus loin dispose encore d'un patrimoine. L'importance des activités réalisées par ces structures avant le conflit justifie l'enthousiasme exprimé en faveur de la relance de cette Organisation. Ce qui a conduit aux experts d'envisager la relance de la CEPGL dès 2006, bien avant la fin de la crise. Les projets tels que celui de la construction du Barrage sur la RUZIZI III, serait une continuité de la politique régionale en matière d'énergie électrique. Ce projet attend encore des financements.

\section{Projet des Bassins Transfrontaliers de Développement ${ }^{37}$}

Les zones de croissance sont des mécanismes «flexibles » qui peuvent être utilisés pour favoriser le développement, la paix et la sécurité38. Elles ont l'avantage d'être généralement non encombrées par les formalités liées aux accords plus conventionnels et codifiés caractérisant les zones de libre échange et des unions douanières. Ces cadres accordent aux sous régions participantes une plus grande flexibilité dans la formulation, l'adoption et la mise en œuvre des politiques du secteur public coordonnées sur une base sous régionale. De même, les investissements frontaliers et la coopération du secteur privé peuvent y être efficacement mis en œuvre. Le choix porté à ce genre de mécanisme pour la région est fondé sur le fait que, partout où une approche industrielle basée sur les ressources a pu s'affirmer, elle a été en grande partie due au développement d'un groupe d'industries en amont et en aval, et des services liés à l'investissement majeur initial ${ }^{39}$. Ce que la région des Grands Lacs ne dispose pas encore actuellement.

De même, la question de la coopération transfrontalière devient de plus en plus importante dans le contexte de la paix, de la sécurité et du développement économique de la Région des Grands Lacs. Les principaux domaines de coopération peuvent inclure premièrement, le développement des institutions, tels que l'établissement d'un Comité des Territoires

36 En ce sens, lire notamment : KABUya kALALA, TShiunza mBIYE François, "Communautés économiques régionales : quelle stratégie d'intégration en RDC? », L'Afrique des Grands Lacs, Annuaire 2009-2010, pp.327-342; Alexandre Taithe, « Les enjeux et effets induits attendus de grands investissements énergétiques : projets hydroélectriques », Observatoire des Grands Lacs, Note $n^{\circ} 6$, novembre 2012, pp.13-14; Nisse Nzereka Mughendi, « Relancer la cepgl en pleine crise économique? L'enjeu du partage des ressources congolaises », s.e., 2009, pp.91-97.

37 Conférence Internationale sur la Région des Grands Lacs, Programme d'Action Régional pour le Développement Économique et Intégration Régionale, Bassins Transfrontaliers de Développement, Projet No. 3.1.2, mars 2006 (Rev. 30 août 2006), 20p.

38 Pour des développements sur la question, lire notamment, Rachid BELKACEM, Moamed BENNOUI, Régis ROUYER, « Un territoire transfrontalier en crise : l'exemple du bassin transfrontalier de LONGWY », Rencontres internationales, Démocratie et management local, Québec, mai 2003, $18 \mathrm{p}$; Delpont, André, « Coopération économique transfrontalière. L'exemple de Lille-Eurométropole franco-belge ", Azkoaga, $n^{\circ}$ 11, 2001, pp.447-466; Association des Régions Frontalières Européennes (ARFE), Possibilités de coopération transfrontalière en Afrique de l'Ouest : Une contribution au processus d'intégration régionale, Rapport final, 22 janvier 2012.

39 Document du projet, op.cit., p.6. 
transfrontaliers ou des Commissions des BTD, tel qu'indiqué dans le Protocole sur la ZSRD, (qui est centré sur la promotion du développement socio-économique en général), les efforts de collaboration entre les agences compétentes de mobilisation d'investissement et l'établissement d'une initiative régionale de réseau de tourisme qui pourrait être connue comme réseau de tourisme de la sous région des Grand Lacs (RTRGL) ${ }^{40}$.

En termes de développement socio-économique, les initiatives principales incluraient le développement des infrastructures de santé et d'éducation ainsi que des infrastructures de télécommunications. Plus spécifiquement, un effort de coopération transfrontalière impliquant trois pays est à même de faciliter le partage des ressources naturelles potentielles pour améliorer la diversité des produits de tourisme ainsi que pour constituer un mécanisme aux termes duquel les ressources et l'expérience sont partagées.

Dans ce cadre, le développement des postes frontières stratégiquement essentiels peut être conçu et mis en œuvre conjointement, afin de s'assurer que le mouvement des marchandises et des services est autant que possible exempt de troubles potentiels et que, celui des marchandises, des services illégaux et des personnes soit correctement contrôlé. De même, puisque les terres limitrophes sous-régionales tendent invariablement de posséder les mêmes ressources naturelles, les avantages et coûts liés au développement de ces ressources, de chaque côté de la frontière, devraient également être invariablement partagés ${ }^{41}$.

Les triangles de croissance ont également des implications sur la paix et la sécurité, ainsi que sur l'intégration sociale et la participation des communautés au développement des régions concernées. Ils favorisent une plus grande coopération et harmonisation des politiques, telles que dans les douanes, les banques et les finances, aux fins de libéraliser le flux des marchandises, des capitaux, et de la main d'œuvre à travers les frontières nationales. Ceci s'applique non seulement au niveau national où les gouvernements essaient de coopérer à réaliser le triangle de croissance, mais cela permet aussi aux autorités locales de formaliser, tout au plus, leurs relations ou, tout au moins, établir des contacts entre eux.

L'idée soutenue en filigrane, est qu'en créant plus de liens d'interdépendance entre les économies locales, la force sera moins susceptible d'être utilisée pour régler un conflit, en raison des effets perturbateurs qu'il aurait sur des activités économiques. Par conséquent, les coûts élevés de la violence favoriseraient la paix ${ }^{42}$. Les secteurs principaux d'activité pour des triangles de croissance incluent (i) la promotion de l'investissement; (ii) la facilitation commerciale (iii) la collaboration du secteur d'affaires et (iv) la planification industrielle.

La promotion de l'investissement vise à explorer les voies pour attirer l'investissement local et étranger dans le triangle de croissance par le marketing, les richesses complémentaires dans la zone, le flux des facteurs de production à travers les frontières, et offrir éventuellement des incitations dans les zones économiques spéciales. Les activités possibles

\footnotetext{
40 Id.

41 Ibid., p.8.

42 Document du projet, p.11.
} 
dans le triangle de croissance incluent notamment des stratégies coordonnées de marketing; le développement des capacités institutionnelles pour les organismes locaux d'investissement; les mesures de coopération inter agences; des approbations harmonisées et des procédures de normalisation pour les projets d'investissement frontaliers; la promotion de l'investissement dans la zone TC appropriée; et un système d'échange de l'information sur l'investissement.

La facilitation commerciale entend examiner les approches visant à améliorer l'efficacité des procédures, de la documentation, et de l'échange de données commerciales dans le triangle de croissance. Les activités possibles incluent notamment l'harmonisation des procédures de douanes et des procédures d'inspections et des exigences requises en termes de documentation, en développant un système cohérent de classification des biens, des systèmes d'information relatifs au commerce, des activités communes de promotion des exportations; en accélérant la question des accords d'échanges commerciaux et établir des normes de qualité à l'exportation pour les produits agricoles.

Dans un contexte de collaboration du secteur d'affaires, les États mettraient en place des mécanismes de coopération entre les secteurs d'affaires dans le triangle de croissance pour soutenir d'autres activités et pour créer une dynamique durable afin de créer l'activité économique. Les activités possibles incluent notamment la création d'un forum d'affaires du Triangle, organiser des sessions de mise en réseau, particulièrement dans les domaines du tourisme et de l'agriculture, et assurer des échanges d'information sur les affaires locales.

En fin, les pays membres s'efforceront à coordonner ensemble une planification industrielle, sur la base des complémentarités et de la richesse potentielle en ressources, en vue de promouvoir un modèle de coopération industrielle à faire profiter à toutes les sociétés dans le triangle de croissance ${ }^{43}$.

Trois pays africains (Ouganda, Kenya et Tanzanie) partageant le Lac Victoria, expérimentent un projet des bassins transfrontaliers de développement. Le projet pilote proposé aux trois pays porte sur les pêcheries et le traitement industriel des poissons. L'objectif poursuivi est de créer un triangle de croissance et fournir la capacité tripartite en vue d'augmenter la valeur ajoutée dans les activités de traitement et la main d'œuvre; planifier le développement d'infrastructures à long terme; mise à niveau des capacités professionnelles et des technologies (...).

Ce projet est conçu pour s'appliquer aux trois pays de l'Est peut être répliqué entre la RDC et ses deux voisins, compte tenu le fait que ces derniers partagent un vaste réseau lacustre qui présentent d'énormes potentialités pour l'industrie de la pêche. 


\section{Projet Régional sur la Sécurité Alimentaire dans la Région des Grands Lacs ${ }^{44}$}

Ce projet est structuré autour de trois composantes ci-après : amélioration de la productivité agricole et des services d'aide aux fermiers; Développement d'établissements piscicoles et sécurité alimentaire et formulation d'un programme sur le développement de l'élevage pour la sécurité alimentaire dans la grande Région des Grands Lacs et de l'Afrique centrale. Son objectif général est de contribuer à la sécurité alimentaire dans la sous région, par le biais du développement des activités de pêche et d'établissements piscicoles, en accordant une attention particulière à la pêche dans les lacs et les fleuves pour contribuer à la réduction de la pauvreté. Le projet prévoit les activités préparatoires visant à mettre au point un programme substantiel pour le développement d'activités de pêche dans la sous région.

Outre la réduction de la pauvreté, le projet devrait permettre aux pays des Grands Lacs de discuter de la coopération régionale en matière de développement de l'élevage et de consulter les partenaires sur la possibilité d'une coopération pour la mobilisation de leur assistance aussi bien technique que financière.

\section{Programme d'amélioration des Projets d'Infrastructures et Facilités de Transport ${ }^{45}$ (Corridor Nord).}

Les pays de la région des Grands Lacs se caractérisent par un enclavement qui a rendu difficile leur intégration physique. Le Programme d'amélioration des projets d'infrastructures de la CIRGL a été élaboré dans la logique qui vise à proposer une plus grande interconnexion des États de la sous-région. Il a été démontré dans plusieurs régions que le développement d'une infrastructure qui augmente ou rende plus facile le contact entre les pays peut stimuler l'intégration productive car elle rapproche des espaces économiques, réduit les barrières physiques au commerce, réduit les coûts de transport et élargit la taille des marchés $^{46}$. Le Programme est structuré autour de quatre axes impliquant la construction ou l'amélioration des insfrastructures de transports dont les routes, les ports intérieurs, les voies navinagables intérieures et l'extension du chemin de fer.

44 Conférence Internationale sur la Région des Grands Lacs, Programme d'Action Régional pour le Développement Économique et Intégration Régionale, Projet Régional sur la Sécurité Alimentaire dans la Région des Grands Lacs, 2006, 37p.

45 Conférence Internationale sur la Région des Grands Lacs, Programme d'Action Régional pour le Développement économique et Intégration régionale, Programme d'amélioration des Projets d'Infrastructures et Facilités de Transport, Projet N . 3.3.1 Corridor Nord, 2006, 24p.

46 Nahuel Oddone, «Coopération régionale dans le domaine de l'intégration frontalière », p.2, disponible en ligne, in www.franceamsud.org/observatorio/images/Biblioteca/integration/coopnahuel.pdf, consulté le 31 mai 2016. 
a) Routes

\begin{tabular}{llll}
\hline Axes routiers retenus pour la RDC & Long/km & Travaux & Coût/EUR \\
Bukavu-Kindu-Kisangani & 1149 & Asphaltage & 130 \\
Bukavu-Uvira & 138 & Asphaltage & 35 \\
Goma-Beni-Kasindi & 441 & Réhab.; asphaltage & 87 \\
Kisangani-Beni & 719 & Asphaltage & 150 \\
Bukavu-Walikali-Lubutu & 417 & Asphaltage & 105 \\
\hline
\end{tabular}

Si dans les pays voisins les efforts ont été fournis, notamment en matière de mobilisation des fonds pour exécuter ce projet, la plupart des routes du Corridor Nord à l'est de la RD Congo sont des routes couvertes d'oyats et en mauvais état ${ }^{47}$. Leur réhabilitation et asphaltage permettrait à ce pays de participer à armes égales à l'ouverture des marchés qu'entrainerait inévitablement la mise en œuvre de multiples accords régionaux auxquels la RDC est liée.

\section{b) Les ports intérieurs}

Le corridor nord entre ces trois pays est relié par les ports de Kisangani et du Lac Tanganyika, mais ces derniers nécessitent des travaux de réhabilitation et autres aménagements. Des études de faisabilité et d'amélioration sont nécessaires pour les ports secs et dépôts intérieurs de conteneurs de trois pays.

c) Voies d'eaux navigables intérieures.

La RDC, le Rwanda et le Burundi sont des pays semi enclavés en ce sens qu'ils dépendent, en grande partie des ports maritimes des pays voisins - à l'exception de l'ouest de la RDC - pour l'importation d'une grande partie de leurs biens manufacturés. Les trois pays sont toutefois reliés par un réseau fluvial et lacustre qui leur offre des possibilités d'interconnexion $^{48}$ au système de transport maritime qui peut relier l'Atlantique à l'océan indien. La réalisation d'une véritable coopération entre eux doit passer par l'intégration des systèmes et les voies des transports des différents pays. En ce sens, la réhabilitation et la navigabilité du fleuve Congo ${ }^{49}$ doivent être conçues en interrelation avec les autres projets d'infrastruc-

47 Projet n³.3.1, op.cit, p.16.

48 Sur les détails sur ces interconnexions lire : Conférence Internationale pour la Région des Grands Lacs (CIRGL), Étude de faisabilité sur la réhabilitation et la navigabilité du fleuve Congo, août 2006, pp.2-5.

49 Pour des détails, lire notamment Camille Ngoma khuabi, « Le régime juridique de navigation sur le fleuve Congo : entre centralisation, application et rejet d'un cadre conventionnel régional mal négocié en RDC, Librairie Africaine d'Études Juridiques, 1(2014), 2014, pp. 355-356, http://www .nomoselibrary.de/index. php? $\mathrm{v}=\mathrm{agb}$. 
tures de transport tels que la route transafricaine Lagos-Mombasa; le réseau routier du Corridor Nord; le Corridor Sud (Projet chemin de fer des Grands Lacs); le Corridor Lobito; le chemin de fer Mombasa-Kisangani, proposés dans le cadre de la CIRGL.

d) Extension des chemins de fer

L'objectif du projet est de relier l'Océan Indien à l'Océan Atlantique par voie ferrée et voies fluviales. Dans ce cadre, il est prévu l'extension du chemin de fer Kenya-Ouganda de Kasese à Kisangani, et la ligne de chemin de fer Kasese-Kisangani avec liens ferroviaires jusqu'à Kigali et Bujumbura. À ce sujet, la TTCA a organisé une réunion extraordinaire à Nairobi en août 2004 afin d'examiner le projet d'extension de la ligne de chemin de fer de Kasese vers la RD Congo, le Rwanda et le Burundi.

Le secrétariat de la TTCA a déjà organisé une réunion d'experts ferroviaires des États membres aux fins de préparer les mandats/cahiers des charges, qui ont été soumis à la Banque de Développement de l'Afrique, avec demande à la banque de financer les études de préfaisabilité. Il reste que les financements nécessaires soient débloqués en vue de sa matérialisation.

\section{Projet de Réhabilitation et d'Inter-connectivité du Barrage d'Inga ${ }^{50}$}

L'énergie électrique constitue l'un des facteurs clés dans le développement économique d'un pays. Son déficit actuel à l'échelle des trois États ralentit l'investissement dans les secteurs productifs, alors qu'il existe en RDC, par exemple, un énorme potentiel non exploité avec de grandes possibilités d'interconnexions. En effet, alors que la capacité totale de toutes les installations hydroélectriques subsahariennes est évaluée à $48000 \mathrm{MW}$, la RDC abrite des potentialités hydroélectriques estimées à $100.000^{51} \mathrm{MW}$ dont $44000 \mathrm{MW}$ concentré au site d'Inga ${ }^{52}$. La capacité actuellement installée est de 2.498,45 MW soit $2,5 \%{ }^{53}$. Une étude de faisabilité sur les travaux de réhabilitation nécessaires au rétablissement de la capacité totale des installations de production et de transmission a été réalisée en

50 Conférence Internationale sur la Région des Grands Lacs, Programme d'Action Régional pour le Développement Économique et Intégration Régionale, Projet de Réhabilitation et d'Interconnectivité du Barrage d'Inga, Projet No. 3.3.7, août 2006, 19 p.

51 Pour des détails lire, George Wolf et Fabrice Lusinde, « Questions Géospatiales et Infrastructures », in Johannes Herderschee, Mukoko Samba (D) et Tshimenga Tshibangu (M) (ed.), Résilience d'un Géant Africain : Accélérer la Croissance et Promouvoir l'emploi en République Démocratique du Congo, Volume III, Sujets transversaux, MEDIASPAUL, Kinshasa, 2012, p.3.

52 VIKA di PANZU, « La réforme du secteur de l'Électricité de la République Démocratique du Congo et le développement des projets de centrales Hydroélectriques Inga III et Grand Inga », communication à la Conférence sur l'hydroélectricité et le développement durable en Afrique, Addis-Abeba, 31 mars -01 avril 2011, disponible www.h4sd.info/getattachment/Documents-and.../ Theme-2_Vika-Di-Panzu.pdf.aspx, 01 octobre 2012.

53 Idem. 
RDC. L'objectif général du projet est de renforcer l'intégration régionale et de promouvoir le développement économique en exploitant le potentiel d'hydroélectricité disponible dans ce pays, pour son utilisation commune par les populations de la Région des Grands Lacs, et en exporter l'excédent vers d'autres régions notamment les régions australes, occidentales et même celles du nord de l'Afrique.

6. Le Projet d'oléoduc régional, (Extension du pipeline de Kampala-Kigali-Bujumbura-RD Congo) $)^{54}$.

La zone de ce projet couvre le principal corridor de transport entre Kampala et la RDC, en passant par le Rwanda et le Burundi. En effet, dans cette région, la majorité des villes sont installées le long de principales voies de transport. Ce qui contribue à une circulation plus dense, nécessaire pour assurer le transport d'autres produits et des personnes vers des zones urbaines. L'installation de l'oléoduc permettra davantage d'attirer de fortes densités démographiques par rapport aux zones plus éloignées de des infrastructures routières.

L'objectif général de l'oléoduc proposé est donc de favoriser l'intégration régionale et de promouvoir la coopération, à travers le développement et l'exploitation commune d'une infrastructure. Il vise également à assurer des moyens rentables et conviviaux du point de vue environnemental, pour le transport des produits pétroliers vers les pays des Grands Lacs $^{55}$ et particulièrement le Rwanda, le Burundi et la RD Congo semi enclavés. En effet, pour satisfaire leurs besoins en matière de produits pétroliers, ces trois pays dépendent de cet oléoduc actuellement limité aux frontières du Kenya. Les pays ont manifesté leur ferme volonté d'étendre cet oléoduc pour ne plus dépendre du transport routier qui est plus onéreux et moins efficace ${ }^{56}$.

La construction de l'oléoduc devrait donc impliquer un engagement politique pour la mobilisation des ressources requises ainsi que pour solliciter la coopération auprès de toutes les parties concernées, afin d'assurer sa réalisation. De même, l'opérationnalisation de l'oléoduc nécessitera, en permanence, le dialogue et la coopération entre les pays impliqués. L'expérience acquise dans le processus de mise en œuvre du projet et, subséquemment de sa gestion, permettra de consolider les relations entre les pays concernés et, partant, promouvoir l'intégration régionale, tout en contribuant à la réalisation de la vision commune définie pour la Région des Grands Lacs.

Il résulte de ce qui précède que, les infrastructures jouent un rôle essentiel en matière de développement économique et de réduction de la pauvreté. Comme l'a bien reconnu l'Union Africaine, des services d'infrastructures bien développés et entretenus notamment dans le domaine d'électricité, des transports, eau et TIC, réduisent les obstacles à la crois-

54 Pour plus de détails, lire : Conférence Internationale sur la Région des Grands Lacs, Programme d'Action Régional pour le Développement Économique et Intégration Régionale, Étude de faisabilité sur le Projet d'oléoduc régional, Projet N³.3.8, mars 2006, 14 p.

55 Idem, p.5.

56 Ibid, p.1. 
sance économique et leurs coûts de transaction et contribuent énormément à améliorer la vie des pauvres en facilitant leur accès aux services publics et sociaux ${ }^{57}$. De mauvaises infrastructures imposent conséquemment des coûts majeurs sur le commerce, en matière de perte de débouchés et d'augmentation des coûts.

Dans les cas de la RDC, du Burundi et du Rwanda, les efforts restent déployer pour la mobilisation des fonds nécessaires à la réalisation des projets proposés pour une plus grande coopération soutenue actuellement par l'Accord-cadre d'Addis-Abeba.

\section{L'Accord-cadre pour la paix, la sécurité et la coopération pour la République démocratique du Congo et la Région ${ }^{58}$, gage d'une coopération économique rénovée pour les trois pays.}

Cet Accord-cadre pour la paix, la sécurité et la coopération pour la République Démocratique du Congo et la Région s'inscrit dans le cadre de la nouvelle doctrine des Nations Unies en matière de résolution des crises dans des régions dévastées par des conflits multiformes.

En effet, après la fin de la guerre froide, plusieurs conflits qui se sont prolongés notamment en Afrique sans qu'aucune réelle explication idéologique ne parvienne à justifier une telle durée, ont conduit les Nations Unies à changer d'approche dans la recherche de leur résolution. Au cours de la même période ont émergé des idées qui combinent l'étude des questions des ressources naturelles et de l'environnement et leurs liens sur la menace à la paix et la sécurité internationales.

De même, les constatations préliminaires d'une analyse rétrospective des conflits internes de ces soixante dernières années conduite par le PNUE relevaient que les conflits liés aux ressources naturelles ont statistiquement deux fois plus de chances de resurgir durant les cinq années suivant la signature d'un accord de paix. Pourtant, moins d'un quart des négociations de paix visant à résoudre de tels conflits ont prêté attention aux mécanismes de gestion des ressources naturelles ${ }^{59}$. Dans le prolongement de ces études, les Nations Unies ont abouti à la conclusion que la gouvernance des ressources naturelles et de l'environnement a une influence déterminante sur la paix et la sécurité et peut donc aussi contribuer à une reprise des hostilités si elle n'est pas correctement gérée dans la période post-conflit ${ }^{60}$. Afin de mieux comprendre et gérer cette nouvelle dimension sécuritaire de l'environne-

57 Union Africaine, Plan d'action pour l'Afrique de l'UA/NEPAD, 2010-2015, Promouvoir l'intégration régionale et continentale en Afrique, 2010, p.1.

58 Voir l'intégralité du texte dans le document S/2013/131.

59 Programme des Nations Unies pour l'environnement, Du conflit à la consolidation de la paix. Le rôle des ressources naturelles et de l'environnement, Nairobi, Kenya, 2009, ISBN : 978-92-807-2957-3, p.5.

60 Programme des Nations Unies pour l'environnement, op.cit., p.5. 
ment, l'ONU dût adopter une approche transversale et a mis en place de politiques structurelles de long terme ${ }^{61}$.

La signature de l'Accord-cadre entre la RDC et la région, s'insère dans cette nouvelle stratégie onusienne de consolidation de la paix dans la période post-conflit. Aussi, en même temps que les efforts diplomatiques se poursuivent, une intense activité de coopération et d'intégration économiques est nécessaire entre la RDC et l'ensemble de ses voisins en vue de soutenir les efforts de paix dans la phase actuelle. Une telle coopération devrait se concrétiser notamment par l'exploitation en commun des ressources naturelles transfrontalières. Tout en contribuant à la consolidation de la paix grâce au développement économique et à la création d'emplois qu'elles favorisent, une gestion concertée des ressources naturelles transfrontalières pourrait ouvrir de nouvelles perspectives pour la consolidation de la paix.

L'Accord a donc pour objectif d'aider à «cerner la cause fondamentale des conflits et mettre un terme aux cycles de violence récurrents» dans l'Est de la RDC et la Région des Grands Lacs.

Quatorze pays de la région des grands lacs l'ont signé. Sans être un pays membre de la région, l'Organisation des Nations Unies prend des engagements au nom de la Communauté internationale. Ces engagements consistent à apporter un soutien à la réalisation des programmes économiques entre la RDC et la région. Il s'agit en réalité de soutenir les projets analysés plus loin, formulés par la CIRGL. Ces engagements ${ }^{62}$ sont exprimés en ces termes :

"Un engagement renouvelé des partenaires bilatéraux à demeurer mobilisés dans leur soutien à la République démocratique du Congo et la région, y compris avec les moyens appropriés pour assurer la durabilité de [ces] actions sur le long terme; et d'appuyer la mise en ouvre des protocoles et des projets prioritaires du Pacte sur la sécurité, la stabilité et le développement dans la région des Grands Lacs ».

"Un engagement renouvelé à travailler à la revitalisation de la CEPGL et à soutenir la mise en ouvre de son objectif de développement économique et d'intégration régionale».

La région des Grands Lacs a également pris un engagement allant dans le sens de renforcer la coopération régionale en ces termes :

61 Pour des détails, lire Ngoma khuabi Camille, L'eau et la stabilité en Afrique centrale. Recherche d'un mécanisme pour une gestion intégrée des bassins transfrontaliers, mémoire de DES, faculté de droit, Université de Kinshasa, 2015, pp.42-47.

62 V. Doc. S/2013/131, p.3. 
"Renforcer la coopération régionale, y compris à travers l'approfondissement de l'intégration économique avec une attention particulière accordée à la question de l'exploitation des ressources naturelles ${ }^{63}$ ».

Il ressort clairement de l'analyse de cet instrument que ses rédacteurs ont bien pris en compte le rôle que les questions environnementales ont joué dans le conflit qui a embrasé toute la région des Grands Lacs africains. L'accord fait ressortir l'importance des ressources naturelles en tant que voies de coopération, de transformation et de consolidation de la paix dans les pays dévastés par la guerre. Il appartiendra, comme rappelé plus loin, à la RDC de choisir parmi la gamme des projets proposés dans le cadre de la CIRGL, ceux qu'elle juge essentiels pour la relance d'une économie formelle à l'intérieur de son territoire, mais dont les activités pourraient avoir des interconnexions avec des projets similaires également financés par la Communauté internationale dans les pays voisins. C'est dire que la Communauté internationale fait peser sur la RDC la charge de déterminer la forme de coopération la plus appropriée qu'elle juge nécessaire pour une intégration économique capable d'endiguer les velléités des crises à répétition à l'Est de son territoire.

Le 31 janvier 2014, les signataires de l'Accord-cadre ont approuvé un plan d'action devant guider la mise en œuvre des engagements décrit dans ce document. Dans le cadre de ce plan d'action, ils ont identifié plusieurs activités-clés à même de soutenir une plus grande coopération régionale l'organisation, dont l'organisation d'une conférence sur l'investissement du secteur privé dans la région des Grands Lacs parmi. Un document sur les opportunités d'investissement ${ }^{64}$ a ainsi été élaboré. L'objectif sous-jacent est d'identifier les projets qui offrent des possibilités de collaboration public-privé, impliquant plusieurs pays, et qui illustrent les opportunités économiques existantes et émergentes dans la région ${ }^{65}$. Sept secteurs, dont l'agriculture, l'énergie, les finances, l'Information, la Télécommunication et la Technologie (ITC), les infrastructures, les mines et le tourisme, ont finalement été identifiés comme secteurs prioritaires compte tenu de leur potentiel de création d'emploi, de stimulation de l'économie et d'impact catalyseur dans la région ${ }^{66}$.

La conférence a finalement été organisée à Kinshasa du 24 au 26 février, par le Bureau de l'Envoyé spécial du secrétaire général des Nations unies pour la région des Grands Lacs et le secrétariat exécutif de la CIRGL, avec le soutien du gouvernement de la République démocratique du Congo. Elle est destinée à offrir notamment aux décideurs politiques et investisseurs, l'occasion de découvrir les opportunités d'investissement qu'offre la région des Grands Lacs, de s'informer au sujet des principaux enjeux régionaux, de discuter de l'évolution de la situation sécuritaire et du climat des affaires et d'étendre leurs réseaux d'affaire. La présence à la conférence des délégations des Nations Unies et de plus hauts responsables

63 Doc. S/2013/131, op.cit, pp.2-3.

64 Nations Unies, Document sur les Opportunités d'Investissement (DOI), processus de consultation, leçons apprises et principales conclusions, mars 2015, 74p.

65 Ibid, p.5.

66 Idem, p.4. 
politiques de pays voisins, de même que des représentants des investisseurs de la région laisse penser à la cicatrisation des méfaits de la guerre et à la possibilité de relancer la coopération économique sur de nouvelles bases.

Il reste à dire que si les différentes résolutions des Nations Unies ont permis de maintenir une situation d'accalmie, il n'en demeure pas moins que la région vit encore une situation à la fois fragile et précaire. Il est urgent que l'ONU tienne ses engagements financiers afin de concrétiser la réalisation des premiers projets transfrontaliers entre la RDC, le Rwanda et le Burundi.

\section{CONCLUSION}

Après les indépendances, l'intégration régionale était perçue comme un moyen devant offrir des possibilités d'expansion du marché, de réalisation d'économies d'échelle et de diversification de la base économique après le morcellement du continent africain. Les accords de coopération et d'intégration économiques conclus pour la mise en place de marchés sous régionaux protégés, offrant un espace économique élargi pour les facteurs de production, la production industrielle et les échanges devraient permettent d'y répondre. La RDC a expérimenté cette forme de coopération d'abord dans le cadre des Accords bilatéraux, ensuite dans le cadre de la CEPGL.

Dans les premiers cas, la coopération s'est révélée plus formaliste, les accords conclus n'ayant prévu aucun mécanisme institutionnel de mise en œuvre. Dans le second cas, la CEPGL, l'Organisation créée à cet effet, a plutôt su développer une forme institutionnalisée plus pratique de la coopération entre les trois États. Les acquis de cette coopération postulent actuellement la relance et/ou l'élargissement ${ }^{67}$ de cette Organisation sous régionale dans un contexte de consolidation de la paix dans cette période post-conflit.

Toutefois, parmi ses faiblesses, l'on note d'abord que le secteur privé n'avait pas été associé aux initiatives de développement devant aboutir à l'intégration des économies. Ensuite, peu de moyens ont été consacrés au développement des infrastructures sous régionales, si bien que ces États restent encore enclavés. Comme pour le reste de l'Afrique, les résultats peu reluisants de premières tentatives d'intégration de la CEPGL peuvent être attribués au faible niveau de complémentarité structurelle des économies nationales. Ce qui a empêché, à son tour, l'accroissement de la production et des possibilités d'échanges ${ }^{68}$, com- $^{2}$ promettant ainsi les avantages attendus de la coopération envisagée.

67 Le professeur Moïse Cifende Kacico propose plutôt l'élargissement de la CEPGL afin d'y inclure les États d'une autre communauté sous régionale, la EAC, pour des détails, lire, Cifende Kacico Moïse, « La CEPGL et le défi de sa revitalisation : plaidoyer pour l'élargissement ou la fusion des deux communautés économiques régionales des grands lacs africains (CEPGL et EAC), pp. 389-445.

68 Banque africaine de développement, Fonds africain de développement, Politique en matière de coopération économique et d'intégration régionale, Février 2000, p.5. 
Actuellement, bien plus qu'avant, la coopération régionale apparaît un puissant levier pour prévenir ou réguler et apaiser les conflits. Philippe Hugon ${ }^{69}$ relève que la réconciliation franco-allemande, l'accord Mercosur visant à éviter la rivalité Brésil/Argentine, les triangles de croissance est-asiatiques, sont autant de moyens qui ont permis de contourner et de neutraliser les rivalités politiques dans ces régions. Les interdépendances entre les intérêts économiques demeurent un moyen de contourner les conflits en puissance souligne l'auteur ${ }^{70}$.

Dans la perspective de la relance de la CEPGL et de consolidation de la paix après deux décennies de conflits meurtriers, les investissements à consentir devront à la fois concerner des projets qui mettent en relation les Gouvernements des États, avec une haute expertise, et ceux qui impliquent directement des populations dans leur réalisation et sollicitant une main d'œuvre plus accrue. Mais cela ne peut être possible sans une réelle coopération politique entre États membres.

Car, comme l'a démontré Franck P., la solidité d'un processus d'intégration régionale dépend d'une part, du degré d'implication des États membres et de leur cohésion politique, et d'autre part, de la pacification des relations interétatiques, auxquelles on peut ajouter la congruence démocratique et l'équilibre géopolitique entre les États membres ${ }^{71}$. Même si l'auteur relativise quelque peu l'importance de la convergence démocratique entre États membres dans la consolidation des processus d'intégration régionale, l'exemple des conflits vécus entre la RDC, le Burundi et le Rwanda, trois États membres d'une Organisation internationale, la CEPGL, démontre plutôt un enchevêtrement complexe des causes mettant face à face déficit démocratique, revendication de citoyenneté et une prépondérance des causes économiques.

S'il est vrai qu'une coopération économique entre la RDC, le Burundi et le Rwanda, axée sur l'exploitation en commun des ressources naturelles transfrontalières, quelle que soit le format envisagé, bilatéral ou multilatéral, à travers notamment la CEPGL ou la CIRGL, peut contribuer à ramener la paix dans cette région, il est tout aussi vrai qu'une telle entreprise ne peut valablement se construire et se pérenniser que si les États membres maintiennent une stabilité politique sur le plan interne. Il est nécessaire que les efforts actuellement entrepris par les Nations Unies en vue de consolider la paix entre la RDC et la région, intègrent la dimension de l'État de droit, gage d'une stabilité politique interne dans ces trois États post-conflit.

69 Philippe Hugon, «L'intégration régionale peut-elle réduire la vulnérabilité? », communication aux journées commémoratives de la Ferdi, 9-10 Janvier 2014, p.11.

70 Idem, pp.11-12.

71 Franck Petiteville, «Les Processus d'intégration régionale, vecteurs de recomposition du système international? », Études internationales, vol. 28, n³, 1997, p.519. 


\section{Indications bibliographiques}

\section{Documents officiels}

Accord commercial entre la République du Burundi, la République Rwandaise et la République du Zaïre, Kigali, le 21 juin 1975.

Accord relatif aux transports aériens entre la République démocratique du Congo et la République Rwandaise, Kinshasa, le 11 septembre 1970.

Accord-cadre pour la paix, la sécurité et la coopération pour la République démocratique du Congo et la Région, 24 février 2013.

Convention culturelle entre les gouvernements de la République du Zaïre et de la République Rwandaise, Kinshasa, le 18 juillet 1972.

Convention d'établissement entre le gouvernement de la République du Zaïre et le gouvernement de la République Rwandaise, Kinshasa, le 18 juillet 1972.

Convention de coopération entre la République du Zaïre et la République Rwandaise pour l'exploitation, le transport et la commercialisation du gaz méthane du Lac Kivu, Bukavu, le 3 mai 1975.

Convention portant Création de la Communauté Économique des États des Grands Lacs (C.E.P.G.L), Journal officiel de la République du Zaïre, $n^{\circ} 20$ du 15 octobre 1980, pp.5-9.

Traité d'amitié et de coopération entre la République du Zaïre et la RépubliqueRwandaise, Kigali, le 28 octobre 1972.

\section{Ouvrages et Articles}

Alain Léon, Ronan Porhel, « La gestion de la multi-appartenance, une nécessité pour poursuivre la stratégie régionale dans l'Afrique des Grands lacs ", Observatoire des Grands Lacs en Afrique, Note $\mathrm{n}^{\circ} 3$, juin 2012.

AlexandRe TAithe, «Les enjeux et effets induits attendus de grands investissements énergétiques : projets hydroélectriques », Observatoire des Grands Lacs, Note nº, novembre 2012.

Banza Malale, Aspects juridiques des crises congolaises. De l'origine à nos jours (1860-2006), éditions PUK, Kinshasa, 2011, 998p.

Cifende Kacico Moïse, « La Cepgl et le défi de sa revitalisation : plaidoyer pour la l'élargissement ou la fusion des deux communautés économiques régionales des grands lacs africains (CEPGL et EAC) ", in BaKandeja Wa Mpungu (dir.), Quelle politique d'intégration pour quelle unité de 1'Afrique du $21^{\text {ème }}$ siècle? Mélanges ndeshyo RURihose, PUK, Kinshasa, 2014, pp.389-445.

Cifende Kасіко Moïse, « Vers la ratification d'une convention sur la libre circulation des personnes, des biens, des services et des capitaux dans la Communauté des Pays des Grands Lacs? », in NDESHYo RURIHOSE (dir.), La République démocratique du Congo : les défis récurrents de décolonisation, de l'État de droit et du développement économique et social, Mélanges Célestin NGUYANDILA, CEDESURK, Kinshasa, 2012.

Delpont, ANDRÉ, « Coopération économique transfrontalière. L'exemple de Lille-Euro métropole franco-belge », Azkoaga, $n^{\circ}$ 11, 2001, pp.447-466. 
Franck Petiteville, «Les Processus d'intégration régionale, vecteurs de recomposition du système international? », Études internationales, vol. 28, n³, 1997, p.511-533.

George Wolf et Fabrice Lusinde, "Questions Géospatiales et Infrastructures », in Johannes Herderschee, Mukoko Samba (D) et Tshimenga Tshibangu (M) (ed.), Résilience d'un Géant Africain : Accélérer la Croissance et Promouvoir l'emploi en République Démocratique du Congo, Volume III, Sujets transversaux, MEDIASPAUL, Kinshasa, 2012, pp.1-95.

Joseph GaHAma, Le Burundi sous administration belge : la période du mandat 1919-1939, Karthala, Paris, 2001.

Kabuya Kalala, Tshiunza Mbiye (F), « Communautés économiques régionales : quelle stratégie d'intégration en RDC? », L'Afrique des Grands Lacs, Annuaire 2009-2010, pp.327-342.

Mabiala mantuba ngoma, « La guerre d'agression de la RDC (1999-2002) : évolution, perceptions et perspectives, in Labana Lokwa (B), La prévention des crises et l'instauration d'une paix durable en RDC, publication de l'Institut pour la Démocratie et le Leadership Politique, Kinshasa, 2002.

Ngoma Khuabi Camille, « Le régime juridique de navigation sur le fleuve Congo : entre centralisation, application et rejet d'un cadre conventionnel régional mal négocié en RDC, Librairie Africaine d'Études Juridiques, 1(2014), 2014, pp.350-382, http://www.nomoselibrary.de/index. php?v=agb.

NGoma kнuabi Camille, L'eau et la stabilité en Afrique centrale. Recherche d'un mécanisme pour une gestion intégrée des bassins transfrontaliers, mémoire de DES, faculté de droit, Université de Kinshasa, 2015, 196p.

NGOMA KHUABI Camille, « Dégradation de l'environnement, épuisement des ressources natu-relles, explosion démographique et changements climatiques : menace sur les frontières ? Regard sur la " doctrine Nguya-Ndila », in NDESHYO RURIHOSE (dir.), La République démocratique du Congo : Les défis récurrents de décolonisation, de l'état de droit et du développement, Mélanges au Professeur Célestin Nguya-Ndila, CEDESURK, Kinshasa, 2012, pp.95-156.

Nisse Nzereka Mughendi, « Relancer la CEPGL en pleine crise économique? L'enjeu du partage des ressources congolaises », s.e., 2009, pp.91-97.

Philippe Hugon, L'intégration régionale peut-elle réduire la vulnérabilité? Communication aux journées commémoratives de la Ferdi, 9-10 Janvier 2014, 15p, disponible en ligne, www.ferdi.fr/sites/ www.ferdi.fr/files/.../10ansferdi_communication_phugon_2.pdf, consulté le 3 mai 2016.

Rachid Belkacem, Mohamed Bennoui, Régis Rouyer, « Un territoire transfrontalier en crise : l'exemple du bassin transfrontalier de LONGWY », in Rencontres internationales, Démocratie et management local, Québec, mai 2003, 18p.

Roland Pourtier, Afrique Noires, $2^{\text {ème }}$ édition revue et augmentée, Hachette, 2010, ISBN : 987-2-01-146141-4.

\section{Autres documents}

Association des Régions Frontalières Européennes (ARFE), Possibilités de coopération transfrontalière en Afrique de l'Ouest : Une contribution au processus d'intégration régionale, Rapport final, 22 janvier 2012. 
Banque africaine de développement, Fonds africain de développement, Politique en matière de coopération économique et d'intégration régionale, Février 2000.

Conférence Internationale sur la Région des Grands Lacs, Bassins Transfrontaliers de Développement, Projet $\mathrm{N}^{\circ} 3.1 .2$, août 2006, 20p.

Conférence Internationale sur la Région des Grands Lacs, Étude de faisabilité sur le Projet d'oléoduc régional, Projet N 3.3.8, mars 2006 (Rev. 30 août 2006), 14 p.

Conférence Internationale sur la Région des Grands Lacs, Programme d'Action Régional pour le Développement Économique et Intégration Régionale. Relance de la Communauté Économique des pays des grands lacs et de ses institutions spécialisées, Projet $N^{\circ} 3.2 .1$, juin 2006 (Rev. 30 août 2006).

Conférence Internationale sur la Région des Grands Lacs, Programme régional sur la Sécurité Alimentaire dans la Région des Grands Lacs, mars 2006 (Rev. 30 août 2006), 37p.

Conférence Internationale sur la Région des Grands Lacs, Programme d'amélioration des Projets d'Infrastructures et Facilités de Transport, Projet N 3.3.1 Corridor Nord, mars 2006 (Rev. 30 août 2006), 24p.

Conférence Internationale sur la Région des Grands Lacs, Projet de Réhabilitation et d'Interconnectivité du Barrage d'Inga, Projet N 3.3.7, octobre 2005 (Rev. 30 août 2006), 19 p.

Nations Unies, Document sur les Opportunités d'Investissement (DOI), processus de consultation, leçons apprises et principales conclusions, mars 2015, 74p.

Programme des Nations Unies pour l'environnement, Du conflit à la consolidation de la paix. Le rôle des ressources naturelles et de l'environnement, Nairobi, Kenya, 2009, ISBN : 978-92-807-2957-3, 44p.

RéPubliQue démocratiQue du Congo, Annexe 4 : Engagements pris au niveau international, Document du Gouvernement congolais présenté à Nairobi à la deuxième réunion du Comité d'Appui Technique, 22-23 juillet 2013.

Union Africaine, Plan d'action pour l'Afrique de l'UA/NEPAD, 2010-2015, Promouvoir l'intégration régionale et continentale en Afrique, 2010. 